\title{
Why have microRNA biomarkers not been translated from bench to clinic?
}

\author{
Kioomars Saliminejad ${ }^{1}$, Hamid Reza Khorram Khorshid ${ }^{2}$ \& Seyed Hamidollah Ghaffari ${ }^{*, 1}$ \\ ${ }^{1}$ Hematology, Oncology \& Stem Cell Transplantation Research Center, Shariati Hospital, Tehran University of Medical Sciences, \\ Tehran, Iran \\ ${ }^{2}$ Genetics Research Center, University of Social Welfare \& Rehabilitation Sciences, Tehran, Iran \\ *Author for correspondence: Tel.: +98 (021) 8490 2665; Fax: +98 (021) 8800 4140; shghaffari200@yahoo.com
}

"Will it be possible to translate miRNA biomarkers from bench to the clinic?"

First draft submitted: 21 October 2018; Accepted for publication: 29 October 2018; Published online: 17 January 2019

Keywords: biomarker $\bullet$ cancer $\bullet$ circulating microRNA $\bullet$ clinical $\bullet$ diagnosis $\bullet$ NGS $\bullet$ plasma $\bullet$ profiling $\bullet$ prognosis - serum

MicroRNAs (miRNAs) are small noncoding RNAs that regulate gene expression by post-transcriptional repression of their mRNA targets [1]. The first evidence of miRNAs involvement in cancer was reported in B cell chronic lymphocytic leukemia in 2002 [2]. Currently, many miRNAs have proved to be dysregulated in many different types of cancers. They can act as oncogenes or tumor suppressor genes, leading to the initiation and progression of malignancies by affecting apoptosis, invasion and angiogenesis [3]. Today, a number of miRNA-targeted therapeutics have reached clinical development, including a mimic of the tumor suppressor miR-34 that is currently in Phase I clinical trials for treating cancer. In addition, antimiRs targeted at miR-122 have reached Phase II trials for treating hepatitis $\mathrm{C}$ infection by downregulation of the replication of hepatitis $\mathrm{C}$ virus RNA genome [4]. After discovery of circulating miRNAs in body fluids including serum or plasma, they were quickly introduced as potential noninvasive diagnostic and prognostic biomarkers in many diseases including cancer [5]. After some promising initial results, it became apparent that the precise quantification of circulating miRNAs was more challenging than expected. So far, in spite of many publications in the past decade, miRNA research has not resulted in validated cancer biomarkers $[5,6]$. The question is why the introduced miRNA biomarkers are not consistent even in a specific cancer. Will it be possible to translate miRNA biomarkers from bench to the clinic?

Numerous reports have been shown that the validation of miRNA biomarkers to be unsuccessful, which could be explained by differences in methodology of the studies, the lack of standard methods for normalization, miRNA processing and the inability to discriminate among closely related miRNAs [7]. Methodological challenges including pre- and postanalytical variables have strong impact on the results and may lead to even contradictory findings [6,7]. Pre-analytical variables are considered as potential causes of inconsistency in miRNA experiments, including samples collection, processing, method of extraction and quality control [8]. The main challenge of post-analytical level is data normalization [9].

The main circulating miRNAs sources are extracellular vesicles that shed into the blood from many cell types. Association of miRNAs with proteins and their small size prevents degradation by RNAses and enhance their stability in blood, making them a good candidate in biomarker research. However, extraction and analysis of miRNAs from these extracellular vesicles may present challenges [10]. During the storage period, miRNAs are released from blood cells and could lead to false results. The results could be significantly different depending on whether plasma or serum is used. Therefore, the protocols should be standardized for sample collection, storage and processing. The commonly used anticoagulants for plasma collection are EDTA, citrate or heparin. It should be noted that some anticoagulants such as heparin inhibits the reverse transcriptase and DNA polymerase enzymes [7]. The level of circulating miRNAs is confounded by many factors such as age, gender, ethnicity, drugs and smoking [9]. Knowing the influence of confounding factors such as age and gender on specific miRNAs levels could help the researchers to better measure the diagnostic or prognostic value of miRNAs in a given context, for example in childhood cancers that belong to a certain age group [11]. 
One of the major factors limiting the use of miRNAs as a diagnostic tool in clinical settings is associated with the fact that frequently reported miRNA biomarkers are detected in patients with different tumor types. For example, the upregulation of circulating miR-21 has been found in patients with colorectal, lung, breast, prostate, liver, esophageal and endometrial cancers [12]. Additionally, the results are not consistent even among very similar studies of the same diseases [13]. Down-regulation of miRNA in tumors might result from genetic alterations or epigenetic silencing, but in circulation, this could happen only if the tumor negatively affected expression of the miRNA(s) in other cells or decreased the stability of the miRNA(s) in circulation. In other words, reductions in circulating miRNAs could be attributed as nonspecific responses to the presence of cancer. Furthermore, tumor burden cannot be correlated with the upregulation of circulating miRNAs. Due to the dilution effects of blood only a small number of tumors, for example late-stage tumors, result in measurable over expression of specific miRNA in the blood. Accordingly, it is more likely that any up-regulation of circulating miRNAs is also the result of response(s) to the presence of cancer [13].

Routine methods for analyzing miRNA expression are qRT-PCR, droplet digital PCR (ddPCR), microarrays and miRNA-seq. miRNA expression by qRT-PCR can be performed in an absolute or relative method. Since no universally invariant calibrator has been found, relative quantification of miRNA in plasma or serum has encountered a serious challenge [14]. Historically, relative expression of miRNAs is mainly relies on small nuclear (e.g., U6) or small nucleolar RNAs (e.g., SNORD44) for normalization. In a study by Masè et al. analysis of the five common reference genes for miRNA research (5S rRNA, U6, hsa-miR-16-5p, SNORD48), demonstrated the poor performance of widely used normalizers U6 [14]. Surprisingly, a range of validated miRNAs reference genes including miR-16, miRs-10b, miR-30a, miR-30d, miR-103, miR-148b, miR-191 and miR-192 have been reported to be differentially regulated in serum or plasma of breast cancer patients in several publications [13].

Consequently, it is essential to verify the expression stability of putative normalizers in each experimental setup [14]. The absolute quantification of miRNAs is advantageous for diagnostic purposes, especially considering that there is no consensus on endogenous controls [7]. miRNAs must be reverse transcribed to cDNA before PCR can be performed, which this step is associated with inherent inefficiencies. ddPCR has shown significant potential as a technology to count miRNA copy numbers at limiting dilutions without bias [15]. Profiling of hundreds of differentially expressed miRNAs from serum or plasma when RNA quantity is limited poses a technical challenge. Pre-amplification of cDNA can overcome this issue; however, it has some disadvantages. Microarrays have a lower sensitivity and specificity than qRT-PCR and are not reliable at a low input level. miRNA-seq is currently the best platform for miRNA discovery, which is extremely sensitive and provides relative expression data for small RNAs in a sample with a greater dynamic range compared with miRNA microarrays [7].

In conclusion, several important considerations are necessary in analyzing circulating miRNAs to find validated cancer biomarkers:

Focusing on extracellular vesicles, improvement of sampling and isolation techniques, in combination with absolute quantification without the use of reference gene(s) may result in practical miRNA biomarker in the future. It should be known which body fluid (serum or plasma) is ideal for the measurement of circulating miRNA concentration. Novel miRNA biomarkers should be further validated in independent studies. It is important to focus on upregulated miRNAs rather than the downregulated miRNAs. Finally, a panel of selected miRNAs, instead of a single one, could be more effective to guarantee that the biomarker is specific to a cancer.

Financial \& competing interests disclosure

The authors have no relevant affiliations or financial involvement with any organization or entity with a financial interest in or financial conflict with the subject matter or materials discussed in the manuscript. This includes employment, consultancies, honoraria, stock ownership or options, expert testimony, grants or patents received or pending, or royalties.

No writing assistance was utilized in the production of this manuscript.

\section{References}

1 Bartel DP. Metazoan MicroRNAs. Cell 173(1), 20-51 (2018).

2 Calin GA, Dumitru CD, Shimizu M et al. Frequent deletions and down-regulation of micro- RNA genes miR15 and miR16 at $13 \mathrm{q} 14$ in chronic lymphocytic leukemia. Proc. Natl Acad. Sci. USA 99(24), 15524-15529 (2002).

3 Oliveto S, Mancino M, Manfrini N, Biffo S. Role of microRNAs in translation regulation and cancer. World. J. Biol. Chem. 8(1), 45-56 (2017). 
4 Rupaimoole R, Slack FJ. MicroRNA therapeutics: towards a new era for the management of cancer and other diseases. Nat. Rev. Drug. Discov. 16(3), 203-222 (2017).

5 Kirschner MB, van Zandwijk N, Reid G. Cell-free microRNAs: potential biomarkers in need of standardized reporting. Front. Genet. 4, 56 (2013).

6 Miotto E, Saccenti E, Lupini L et al. Quantification of circulating miRNAs by droplet digital PCR: comparison of EvaGreen-and TaqMan-based chemistries. Cancer Epidemiol. Biomarkers. Prev. 23(12), 2638-2642 (2014).

7 Moldovan L, Batte KE, Trgovcich J et al. Methodological challenges in utilizing miRNAs as circulating biomarkers. J. Cell. Mol. Med. 18(3), 371-390 (2014).

8 Gustafson D, Tyryshkin K, Renwick N. microRNA-guided diagnostics in clinical samples. Best Pract. Res. Clin. Endocrinol. Metab. 30(5), 563-575 (2016).

9 Singh R, Ramasubramanian B, Kanji S et al. Circulating microRNAs in cancer: hope or hype? Cancer Lett. 381(1), 113-121 (2016).

10 Vickers KC, Palmisano BT, Shoucri BM et al. MicroRNAs are transported in plasma and delivered to recipient cells by high-density lipoproteins. Nat. Cell. Biol. 13(4), 423-433 (2011).

11 Keller A, Meese E. Can circulating miRNAs live up to the promise of being minimal invasive biomarkers in clinical settings? Wiley Interdiscip. Rev. RNA 7(2), 148-156 (2016).

12 Pogribny IP. MicroRNAs as biomarkers for clinical studies. Exp. Biol. Med. (Maywood) 243(3), 283-290 (2018).

13 Witwer KW. Circulating microRNA biomarker studies: pitfalls and potential solutions. Clin. Chem. 61(1), 56-63 (2015).

14 Masè $\mathrm{M}$, Grasso $\mathrm{M}$, Avogaro $\mathrm{L}$ et al. Selection of reference genes is critical for miRNA expression analysis in human cardiac tissue. A focus on atrial fibrillation. Sci. Rep. 7, 41127 (2017).

15 Stein EV, Duewer DL, Farkas $\mathrm{N}$ et al. Steps to achieve quantitative measurements of microRNA using two step droplet digital PCR. PLoS ONE 12(11), e0188085 (2017). 
